\title{
Desafios da Covid-19 nas empresas: Breve análise de desempenho do marketing digital
}

\author{
Daniela Neves ${ }^{1}$, Silvia Fernandes ${ }^{2}$ e Fátima Carvalho ${ }^{3}$ \\ ${ }^{1}$ Faculdade de Economia, Universidade do Algarve, Portugal | danniela.seven@gmail.com | \\ https://orcid.org/0000-0000-0000-0000 \\ ${ }^{2}$ Faculdade de Economia \& Cinturs, Universidade do Algarve, Portugal | sfernan@ualg.pt | \\ https://orcid.org/0000-0002-1699-5415 \\ ${ }^{3}$ Faculdade de Ciências Humanas e Sociais \& Cinturs, Universidade do Algarve, Portugal | \\ flcarvalho@ualg.pt | https://orcid.org/0000-0001-6925-981X
}

\begin{abstract}
Resumo: O marketing surgiu para ampliar a relação entre o consumidor e a empresa. Mas para resistirem no contexto atual, as empresas devem aumentar as suas ações estratégicas de marketing digital. Isso requer especificidade, o que ressalta a importância das métricas e monitoramento, que consiste em avaliar os resultados obtidos dessas ações. Este estudo compara as respostas de importantes empresários da região (Algarve), no setor do marketing digital e TIC, a um questionário quanto ao seu desempenho em termos de media social. Da análise qualitativa feita (em Nvivo), sobre o conteúdo obtido, denota-se uma 'maturidade' diferenciada em termos das suas perceções dos clientes pelas redes sociais. Alguns revelam um estádio menos maduro, i.e., em termos das métricas de media social usadas no sentido de melhorarem as suas estratégias no atual contexto de mercado. Nesse sentido, estes denotam uma maior preocupação com conteúdos e soluções económicas do que com conteúdos e alterações focadas em produtos chave. A escolha dos KPI certos, a integração de canais e o recurso a influencers são práticas a explorar mais.
\end{abstract}

Palavras-chave: Marketing Digital; Media Social; Métricas; Análise Qualitativa.

\section{Covid-19 Challenges in Business: Brief Analysis of Digital Marketing Performance}

\begin{abstract}
Marketing emerged to expand the relationship between the consumer and the company. But in order to resist in the current context, companies must increase their strategic actions of digital marketing. This requires specificity, which highlights the importance of metrics and monitoring, which consists of evaluating the results obtained from those actions. This study compares the responses of important entrepreneurs in the region (Algarve), in the sector of digital marketing and ICT, to a questionnaire regarding their performance in terms of social media. From the qualitative analysis carried out (in Nvivo), on the content obtained, there is a different 'maturity' in terms of their perceptions of customers through social networks. Some reveal a less mature stage, i.e., in terms of social media metrics used to improve their strategies in the current market context. In this sense, these show a greater concern with content and economical solutions than with content and changes focused on key products. Choosing the right KPIs, integrating channels and using influencers are practices to further explore.
\end{abstract}

Keywords: Digital Marketing; Social Media; Metrics; Qualitative Analysis.

\section{Introdução}

Esta época atual está a marcar a história do mundo, não somente devido ao impacto da pandemia Covid-19 e a crise resultante, como também pela transformação digital que as pessoas e as empresas estão a passar. Muitos empresários reportam grandes prejuízos resultantes da diminuição das suas vendas de produtos e serviços, devido ao confinamento social. Em busca de soluções, aumenta o interesse das empresas em marketing digital e outsourcing desta valência (Negócios SC, 2020), reconhecendo a importância dessa valência para a preservação e competitividade do seu negócio.

O e-commerce possibilitou que este tipo de marketing se expandisse, devido ao crescimento das vendas online e da expansão do público que usa a internet. A utilização do marketing digital por uma organização atualmente possibilita que esta expanda o seu número de clientes para comercializar o seu produto/serviço online (Cruz \& Silva, 2014). 
Envolve uma abordagem de marketing que utiliza os canais eletrónicos para enriquecer a relação entre as organizações e os clientes (que podem ser outras empresas). É um conceito amplo, que descreve o marketing de produtos ou serviços que utilizam tecnologias digitais, incluindo publicidade display em telemóveis (ou outro meio digital). Difere do marketing convencional por envolver o uso de canais e métodos que permitem a uma empresa analisar campanhas de marketing e entender o que está a funcionar (e o que não está) de forma rápida e autêntica (em qualquer momento e lugar).

\section{Oportunidades do Marketing Digital}

O conceito de marketing digital abrange todas as plataformas digitais e tecnologias modernas em interação, execução e controlo de estratégias de marketing para uma maior satisfação do cliente (Yamin, 2017). O uso da internet, das redes sociais, das apps (aplicações móveis) e de outras comunicações digitais tornou-se parte da vida diária de muitas pessoas. A taxa atual de uso da internet entre adultos é alta, sem contar com a da próxima geração de consumidores que é ainda maior (falando dos jovens).

No presente contexto de pandemia, cada vez mais estamos ligados através das redes sociais (entre outros tipos de plataformas). O seu uso crescente cria novas oportunidades para os técnicos de marketing atraírem clientes. As empresas podem beneficiar do marketing digital, incluindo a otimização de busca (SEO), otimização de marketing (SEM), marketing influenciador, automação de conteúdo, marketing de campanha, marketing de redes sociais, marketing direto por e-mail, publicidade display, e-books e jogos (Bala \& Verma, 2018).

Com os rápidos desenvolvimentos tecnológicos, o marketing digital mudou o comportamento de compra dos clientes (em termos de modos de pagamento, interação, etc.) e trouxe várias novidades para os consumidores, como descrevemos abaixo:

\section{-Atualização de produtos/serviços}

As tecnologias de marketing digital permitem aos consumidores informações da empresa sempre atualizadas. Hoje em dia, muitos consumidores usam internet sem fios (wireless) a partir de qualquer lugar, pelo que as empresas devem atualizar continuamente as suas informações.

\section{-Aumento do envolvimento}

Com o marketing digital, os consumidores podem envolver-se mais com as diversas atividades da empresa: podem visitar o site da empresa, ler informações sobre os seus produtos/serviços, fazer compras online, fornecer feedback, criar conteúdo, etc.

\section{-Informação clara sobre produtos/serviços}

Um site 'alimentado' pelas redes sociais fornece informações abrangentes sobre os produtos, nas quais os clientes podem confiar e tomar decisões de compra.

\section{-Comparação fácil com outras empresas}

Como muitas empresas promovem os seus produtos/serviços usando o marketing digital, os clientes podem fazer comparações entre produtos de diferentes fornecedores em termos de custo e tempo. Não precisam assim de visitar uma série de diferentes pontos de venda para adquirirem conhecimento sobre produtos/serviços de interesse.

\section{-Compras em tempo real}

Uma vez que a internet está disponível, não deve existir restrição de tempo para quando comprar um produto/serviço online. 


\section{-Compartilhar o conteúdo de produtos/serviços}

O marketing digital dá a oportunidade de partilhar o conteúdo do produto/serviço com outras pessoas. Usando a rede digital, pode-se facilmente transferir informações sobre as características de um produto/serviço para outros.

\section{-Preços claros}

A empresa mostra os preços dos seus produtos/serviços através do canal digital, o que torna os preços claros para os clientes. E a empresa pode alterar regularmente os preços.

\section{-Possibilidade de compra instantânea}

Atualmente, os consumidores podem facilmente obter muitas informações online antes de tomarem decisões de compra. Têm acesso a fornecedores em qualquer parte do mundo e também têm acesso aos preços de mercado atualizados em tempo real.

O marketing digital é uma ferramenta fulcral no desempenho atual dos negócios. O uso de técnicas de marketing eletrónico tem conduzido a uma evolução das técnicas tradicionais de marketing para ambiente digital (García et al., 2019).

\subsection{Papel das Redes Sociais}

As redes sociais foram definidas como estruturas dinâmicas e complexas constituídas por pessoas com valores ou objetivos comuns, interligadas de modo horizontal e predominantemente descentralizado. Podem surgir em torno de objetivos diversos como: políticos, económicos, culturais, informacionais, entre outros. Por sua vez, a análise das redes sociais é usada para explicar uma série de fenómenos caracterizados pela troca intensiva de informação e conhecimento entre pessoas ou grupos (Souza \& Quandt, 2008). Estas redes podem assumir diferentes formatos e níveis de formalidade no decorrer do tempo.

A velocidade das mudanças e a necessidade constante de inovação nos negócios são fatores-chave na emergência e visibilidade que as redes sociais têm alcançado. Outros autores caracterizam as redes como estruturas abertas, possibilitadas de se desenvolver de modo ilimitado, integrando um conjunto de "nós" que se comunicam com a rede por meio de códigos de comunicação semelhantes (Alméri et al., 2013). As redes sociais são redes de comunicação que envolvem uma linguagem simbólica, limites culturais, relações de troca e de poder. Surgiram nos últimos anos como um novo padrão organizacional capaz de expressar, através da sua arquitetura de relações, ideias de caráter inovador, com a missão de ajudar a lidar com alguns dos desafios atuais (Fialho, 2014).

Atualmente as redes sociais são descritas como uma tecnologia moderna, que facilita a criação de conteúdo e a interação online. Uma classificação de redes sociais pode incluir: projetos colaborativos, blogues, comunidades de conteúdo (exo: YouTube), sites de redes sociais (exº $\mathrm{s}$ : Facebook, Twitter), mundos de jogos virtuais e mundos sociais virtuais (Hysa \& Spalek, 2019). No entanto, dada esta variedade, estão incluídas no conceito mais abrangente de media social. A adaptação às redes sociais gerou mudanças importantes para as empresas e sociedade, disponibilizando um amplo centro de conhecimento prático e de maior alcance, atendendo vários níveis de mercado. Estas redes passaram a criar tendências comportamentais no dia-a-dia dos indivíduos e empresas.

Estudos consideram as redes sociais como um mecanismo de marketing eficaz, por terem um impacto significativo na capacidade das empresas em desenvolver/manter a lealdade dos clientes e comportamentos de compra (Ceyhan, 2019). Atualmente, estão conscientes desta importância, pelo que implementam planos de marketing de redes sociais, i.e., integram as redes sociais nos seus planos de marketing. 


\section{Desempenho e Co-Criação}

Um potencial da média social e outros ambientes digitais de interação é a co-criação. Uma das abordagens que orientam o processo de co-criação por meio da média social envolve os seguintes conceitos (Ramaswamy, 2008):

Diálogo: as empresas devem desenvolver diálogos significativos com os consumidores; Acesso: as empresas devem proporcionar aos clientes acesso para desenvolver um diálogo desse tipo;

Relação risco-retorno: as empresas devem considerar como gerir o risco e a relação de retorno, tanto para o cliente como para si próprias; e

Transparência: a informação deve ser partilhada de modo transparente.

Os benefícios da implementação de redes sociais aumentam a consciencialização para a melhoria do relacionamento com os clientes. Este aspeto contribui para aumentar a capacidade de alcançar clientes em escala global. Para melhorar o posicionamento competitivo das empresas, a maioria delas precisa monitorar e analisar cuidadosamente tanto o conteúdo gerado pelo cliente nas suas redes sociais, como nas dos seus concorrentes. O objetivo é encontrar, a partir desses dados, a informação necessária e usá-la para análise e previsão de tendências. Também o feedback dos clientes gera um grande conjunto de dados que será necessário analisar de modo a melhorar o desempenho dos serviços da empresa (Pourkhania et al., 2019).

Pesquisas recentes revelam novas tendências comportamentais dos clientes, como é o caso da crescente procura por produtos personalizados e a disposição dos clientes para se envolverem ativamente no desenvolvimento de produtos. Tal envolvimento influi nas práticas de marketing a nível estratégico e tático. Muitas empresas já oferecem produtos que podem ser personalizados de acordo com os desejos do consumidor final, estando atentas à criação de condições que permitam a colaboração dos clientes no desenvolvimento e teste de novos produtos (co-criação).

O site é um dos elementos-chave da estratégia de marketing digital. As empresas devem basear o seu design e conteúdo nos objetivos de negócio e nas necessidades do mercadoalvo, com ênfase na sua usabilidade e acessibilidade. Por sua vez, as redes sociais associadas são um elemento-chave no processo de captação do comportamento do consumidor (Sormaz et al., 2019). Utilizando as redes sociais, as empresas podem abordar os seus clientes de modo mais natural e informal, apresentando-lhes histórias autênticas sobre as suas marcas.

\subsection{Potencial do Multi-Canal}

Depois de consideradas as tendências, a questão é como usá-las e integrá-las, aproveitando o seu novo potencial. É importante considerar o futuro das redes sociais nesse contexto, uma vez que são um canal vital de marketing e comunicação para empresas e organizações. Isto deve-se à constante inovação que ocorre, tanto do lado tecnológico (plataformas que acrescentam novas funcionalidades e serviços), como do lado do consumidor (pessoas e marcas que encontram novos usos nas redes sociais). Além disso, são culturalmente significativas uma vez que se tornaram, para muitos, o domínio primário no qual recebem importantes quantidades de informação e partilham aspetos das suas vidas.

Mais de $15 \%$ das pesquisas feitas online são sobre coisas novas, i.e., novos produtos, novos problemas, novas preferências (Cardoso, 2020). E com a media social cada vez mais variada, o 'percurso' do consumidor, i.e., os pontos de contacto que usa para as suas necessidades (como o Youtube, Gmail, Instagram, etc.) é mais variado. Daí que seja imperativo dominar estes vários canais, suas dinâmicas e sua integração. 
$\mathrm{O}$ atendimento ao cliente baseado nas redes sociais tende a tornar-se mais personalizado e ubíquo (acompanha o cliente). Os clientes são capazes de se envolver com as empresas em qualquer lugar e a qualquer hora. Assim, as soluções para os problemas dos clientes serão ainda mais acessíveis e imediatas, utilizando abordagens multi-canal. E daí, procurar dar resposta antes mesmo que o cliente perceba um problema ou tenha uma pergunta (Appel et al., 2019).

Dada a relevância crescente desta área, decidimos analisar qualitativamente (em termos de conteúdo e referências nesse conteúdo) as respostas a um breve questionário, dirigido a especialistas da região (Algarve), na área do marketing digital e TIC, a fim de:

- Auscultar na linguagem usada diferentes graus de 'maturidade' quanto à gestão de marketing e media digital no contexto pandémico que atravessamos.

\section{Breve Análise Qualitativa}

É primordial aproveitar o manancial de media social existente para as empresas retirarem um maior retorno dos seus investimentos em marketing. Este trabalho pretende contribuir para tal, pelo aporte de ilações retiradas de uma análise qualitativa das respostas a um questionário a sete empresários da região na área do marketing digital. Esse instrumento assenta nas questões apresentadas na tabela 1, e foi dirigido por email a essa amostra de empresas no sentido de atuarem como 'painel de especialistas'. O objetivo é comparar o que os seus responsáveis sentem e perspetivam em termos de desempenho das suas redes sociais especialmente no contexto atual.

Tabela 1. Questões (e sub-questões) consideradas

O objetivo deste questionário é saber, a partir do que a empresa ausculta do papel das redes sociais no seu marketing digital, se mede o que se segue e que ilações tira disso:

1) a) Dos comportamentos auscultados nas redes sociais, fizeram alterações aos produtos e serviços? b) Porquê? c) Quais?

2) a) Além de gostos e cliques, houve conversões, isto é, venderam mais? b) Em que situações? c) em quanto aumentou o vosso retorno (\%)?

3) a) Analisam padrões de perceção dos utilizadores quanto às vossas ações de marketing digital? b) Em que KPI (indicadores-chave de desempenho) se focam agora a partir desse conhecimento?

4) a) Qual a \% de outsourcing de marketing que fazem a influencers? b) Aumentou?

5) a) Fazem integração de canais (Youtube com Instagram com Facebook com website...) para captar os pontos de contacto por onde "anda" o cliente? b) Que estratégias têm sobre isso?

Pretende-se mostrar a importância de incluir a visão de especialistas em estudos sobre fenómenos multideterminados (Pinheiro et al., 2013). Nessa aceção, o especialista representa uma perspetiva específica sobre o assunto, a ser integrada com outras visões sobre o tema. Na secção seguinte, apresentam-se os resultados de uma análise ao conteúdo das respostas obtidas, usando o software Nvivo v12. As 7 empresas envolvidas são: Growme - 3 responsáveis responderam; e Dengun, Algardata, Van e Visualforma - 1 responsável de cada uma destas respondeu. Estes respondentes são responsáveis pelo departamento de marketing. O questionário foi enviado a outras empresas que, entretanto, não responderam.

No caso da Growme, responderam três colaboradores porque este artigo resultou de um relatório de estágio (de 2 meses) de uma das autoras nessa empresa. Aí, o questionário foi feito aos dois sócios gerentes e a um funcionário responsável pela gestão de clientes. 
Houve dois motivos principais para a escolha destas empresas, nomeadamente das restantes - Algardata, Visualforma, Van e Dengun: 1) a sua proximidade à empresa onde decorreu o referido estágio (Faro e arredores); e 2) a popularidade destas empresas (empresas líderes de mercado).

\subsection{Codificação das Respostas}

No Nvivo, a codificação é uma das várias técnicas para tirar sentido aos dados disponíveis. O modo de gerir a codificação depende do desenho de investigação usado. Codificar o conteúdo da amostra em tópicos (temas) permite constituir nós. Isso é fundamental na maioria dos projetos qualitativos, pois organiza o material reunido em temáticas gerais. Ao abrir um nó no Nvivo, é mostrado todo o seu conteúdo que pode incluir: textos, audio, vídeos, imagens, etc. (que foram codificados nesse nó). Um nó pode ainda ser ligado a um campo memo que pode descrever aspetos a analisar aí, ou até ser recodificado num outro nó. Por outro lado, os casos são as respostas das empresas objeto de estudo.

Inquiridos os sete empresários (do painel), sobre mudanças no comportamento relativo às redes sociais durante a pandemia, segue-se um gráfico que mostra as referências que eles fizeram aos tópicos (nós) correspondentes às questões enviadas.

Gráfico 1. Número de referências aos tópicos do questionário

\section{Tópicos das questões ao painel de especialistas}

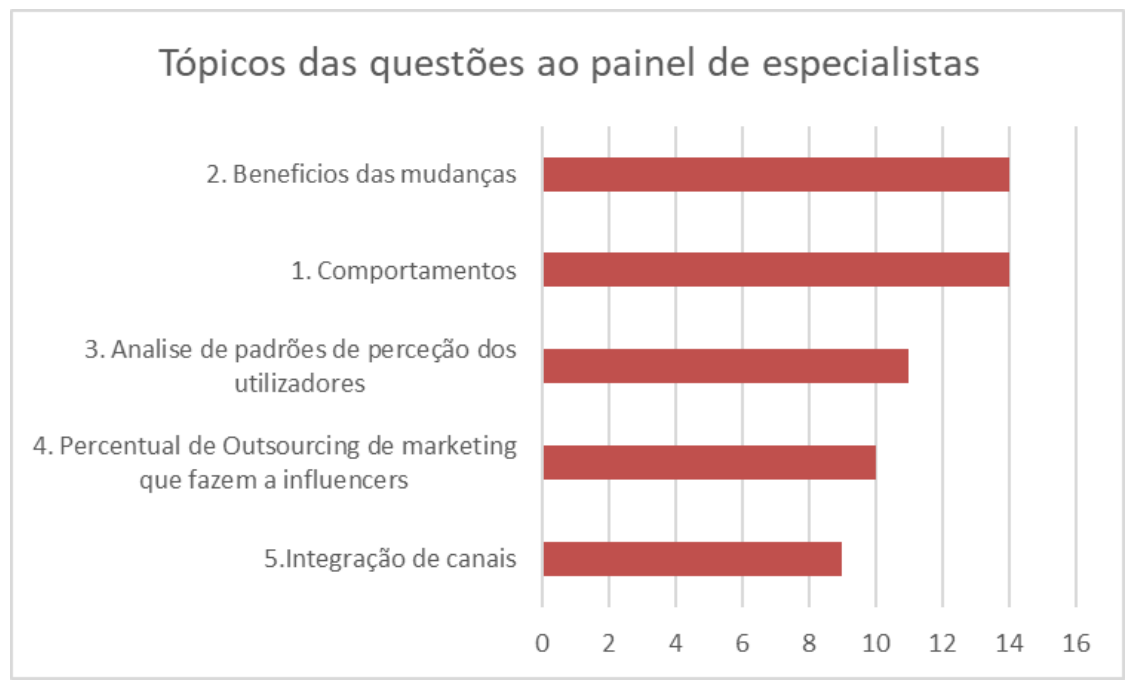

O gráfico mostra que o maior número de referências está associado aos tópicos (temas) sobre comportamentos (alterações de produtos/serviços) e benefícios das mudanças (vendas, retorno). A seguir, vem a análise da perceção dos clientes (reações nas redes sociais e Kpi - indicadores de desempenho/métricas). Tais referências, ou seja, quantos dos inquiridos se referiram a certo tópico, não significam que as respetivas empresas o façam na prática. Indicam que falaram disso e reconhecem a importância desse aspeto. Por isso, prosseguiu-se a análise no sentido de incluir mais outputs, nomeadamente as nuvens de palavras e sua comparação com os diálogos dos respondentes. 


\subsection{Conteúdo e Linguagem Usada}

Complementando os diálogos com as 'nuvens' de palavras (word clouds) obtidas (tabela 2), constata-se que: a Growme incide mais no papel do marketing digital nas vendas e soluções; a Algardata e a Visualforma incidem mais nos produtos e conversões; a Dengun nas leads ${ }^{1}$ e conversões; e a Van nos produtos e alterações. Um aspeto comum é o facto de todas referirem que o seu marketing digital aumentou.

Tabela 2. Frequência de termos das nuvens de palavras

\begin{tabular}{ll}
\hline \multicolumn{1}{c}{ Empresa inquirida } & \multicolumn{1}{c}{ Frequências maiores (e menores) de termos usados } \\
\hline Growme (Cláudio Barbosa) & $\begin{array}{l}\text { Marketing, aumentou, outsourcing, Facebook, pandemia } \\
\text { (comportamentos, conversões, analisar) }\end{array}$ \\
Growme (Frederico Honrado) & $\begin{array}{l}\text { Marketing, aumentou, serviços, clientes, vendas (gostos, } \\
\text { conversões, analisar, auscultar) }\end{array}$ \\
Growme (Ivo Pontes) & $\begin{array}{l}\text { Marketing, aumentou, site, soluções (comportamentos, } \\
\text { influencers, conversões, integração, alavancar) }\end{array}$ \\
Algardata (Juan Correia) & $\begin{array}{l}\text { Marketing, aumentou, produtos, conversões, alterações, Youtube } \\
\text { (influencers, campanha, captar, indicadores, canais, cliques) }\end{array}$ \\
Dengun (Maxine Leal) & $\begin{array}{l}\text { Marketing, aumentou, leads, journey, hubspot, alterações, } \\
\text { conversões, cliques (canais, campanha, captar, colaborar, } \\
\text { commerce) }\end{array}$ \\
Van (Pedro Rosa) & $\begin{array}{l}\text { Marketing, aumentou, produtos, comportamentos, alterações, } \\
\text { redes (canais, influencers, serviços, indicadores, perceção, } \\
\text { integração, performance) }\end{array}$ \\
& Marketing, aumentou, produtos, comportamentos, redes, \\
& conversões, retorno (perceção, serviços, integração, indicadores, \\
performance, outsourcing, influencers)
\end{tabular}

Claudio Barbosa (Growme) concordou que houve mudança de comportamento nas redes sociais durante a pandemia, o que implicou alterações em produtos e serviços. "Com a pandemia, decidimos colocar posts que demonstrassem o comportamento do consumidor durante esta fase, e alertar para a importância do marketing digital".

Frederico Honrado (Growme) também verificou mudanças de comportamento nas redes sociais tais como: "criámos soluções de web design mais económicas". A razão para tais mudanças foi: "pensamos que o poder de compra possa ter sido reduzido. Havendo quebra de rendimentos, pensamos que o dinheiro disponível para os nossos serviços é inferior".

Ivo Pontes (Growme) também observou alterações de comportamento nas redes sociais: "porque queremos estar em linha com as necessidades dos clientes.. adaptámos a linguagem das soluções para que as pessoas nos percecionem como especialistas em determinadas áreas".

Juan Correia (Algardata) considerou que as mudanças foram pontuais e, portanto, dependeram de determinados fatores: "fizemos algumas alterações sobretudo com foco nos produtos que tiveram mais atenção".

${ }^{1}$ Termo que descreve o início do interesse de um possível cliente num dado produto/serviço da empresa. Como por exº: o preenchimento de um inquérito no site, subscrição de um e-book, etc. As leads referemse a conversões que ainda não resultaram numa venda, mas que são importantes de monitorar. A conversão é o que se espera alcançar. Por exº , quando a pessoa solicita um orçamento por entrar na base de leads (que lhe dá acesso às publicações de newsletters). Estas métricas ajudam a entender o alcance de uma campanha e avaliar a eficácia das estratégias utilizadas. 
Entretanto, Maxine Leal (Dengun) também detetou mudanças comportamentais: "fizemos essencialmente alterações ao nível das estratégias implementadas, indo ao encontro das novas necessidades do mercado". Por sua vez, os responsáveis da Van e da Visualforma referem também alterações nos produtos ajustadas às necessidades atuais dos clientes.

Assim como o estudo de Mason et al. (2021), os resultados obtidos (tendo em conta a tabela 2 dos termos mais e menos usados e as 'narrativas' dos responsáveis) mostram que no contexto atual os consumidores aumentaram a sua utilização da media social como ferramenta para saber informações de produtos, avaliá-los e fazer compras online. Isso demonstra a importância crescente do marketing digital. Este estudo pode servir para ajudar as empresas a perceber mudanças nos comportamentos dos consumidores ao nível das redes sociais, e daí repensar a abordagem do seu marketing digital.

\subsection{Benefícios das Mudanças}

Nas narrativas dos respondentes (suas respostas), Claúdio Barbosa (da Growme) detetou que "houve mais vendas com o novo website, mas que devido à pandemia outros clientes acabam por cancelar contratos". Assim, as vendas sofrem um impacto negativo: "acabou por não haver aumentos significativos".

Frederico Honrado, da mesma empresa, notou que "a grande maioria dos nossos clientes venderam menos". Porém, Honrado lembrou "um cliente que tem uma loja online de papel decorativo de parede que vendeu mais aumentou as suas vendas em cerca de $100 \%$ ".

E ainda Ivo Pontes detetou benefícios da mudança de marketing, porém não teve como provar a melhoria através de dados concretos.

Juan Correia (da Algardata) confirmou que "as conversões aconteceram sobretudo em produtos para responder à pandemia Covid-19". Referiu dados sobre a melhoria: "a percentagem de aumento das vendas foi de aproximadamente $10 \%$ ".

Maxine Leal (da Dengun) ponderou que a melhoria nas vendas "depende da área de negócio". Explicou que "durante a pandemia sentimos naturalmente redução de campanhas relacionadas com os setores do turismo e hotelaria. Paralelamente, verificamos aumento de conversões de plataformas e-commerce".

Em relação a métricas de desempenho de redes sociais, Ana Parra (da Visualforma) refere que "usamos os KPI comuns" não especificando quais. E acrescenta "não fazemos integração de canais pois ainda não os ligámos". Quanto à empresa Van, Pedro Rosa diz "focamo-nos no alcance, total de interação, média de interação por publicação e \% bruta de crescimento de cada mês". Refere ainda que faz integração de canais e recorre a influencers.

Ao comparar estes diálogos com as nuvens de palavras denota-se uma diferenciação na linguagem e métricas usadas. Essa variedade revela uma maior incidência nos serviços/ site pela Growme e Visualforma e nas conversões/leads pelas outras empresas (Algardata, Van e Dengun). Ou seja, denota-se uma 'maturidade' crescente de umas para as outras. A linguagem e resultados das últimas transmitem um nível mais 'avançado' em termos de gestão das redes sociais. Pois referem métricas mais avançadas de desempenho e refletem estratégias novas a partir da sua aplicação. Tais estratégias incluem a integração de canais e o outsourcing de influencers. Estas práticas têm uma importância crescente pois com o nível de exigência e rapidez de resposta atualmente requeridos, o marketing digital in-house (interno) pode ser insuficiente.

\section{Conclusão}

Constata-se que o marketing digital e as redes sociais são ferramentas que se conjugam cada vez mais, perante os desafios atuais em que o online é fulcral nos relacionamentos sociais e económicos. 
As empresas inquiridas, usando uma análise qualitativa (do conteúdo das suas respostas), revelam uma 'maturidade' diferenciada face à sua performance de media social. Denotase na Growme, comparada com as suas concorrentes (líderes de mercado), uma estratégia mais adaptativa explorando menos métricas. Nas outras empresas (especialmente na Dengun e Van) uma estratégia mais pró-ativa.

Ter milhares de seguidores (gostos, cliques) pode ser irrelevante se eles não se envolvem com as marcas e seus produtos (engagement). Nesse caso, os esforços de uma empresa nesta área não estão a ser direcionados no seu maior potencial. Os indicadores de maior envolvimento são partilhas, comentários, leads e conversões. Esta base é que potencia consumir conteúdo no sentido de passar para o consumo de bens/serviços, gerando aqui conversões (vendas, pedidos, reservas, etc.). A monitorização deste envolvimento e a consequente inovação dos produtos ajuda a dar as respostas que os consumidores realmente procuram.

Essas formas de envolvimento ativo é que permitem às empresas terem um maior retorno em termos do seu desempenho de media social. Tal abordagem ajuda a repensar o tipo de conteúdos que as empresas publicam nos seus canais. Daí que deva existir um plano de marketing digital que inclua métricas específicas. As redes sociais são realmente uma fonte valiosa, de baixo custo e de 'voz' dos clientes, permitindo que as empresas afinem as suas atividades de marketing digital. Podem ser usadas como 'relações públicas' e ferramentas promocionais, ou como instrumentos que permitem aos clientes personalizar a sua experiência online e os produtos que adquirem (co-criação). Isso ainda se intensifica mais por meio da media móvel (dispositivos móveis e apps relacionadas). Os gestores devem estar conscientes deste manancial e das estratégias de comunicação subjacentes, a fim de melhorar a sua experiência digital. Devem estar atentos aos avanços tecnológicos, como as media digitais, para melhorar a sua abordagem de marketing (Junusi, 2020).

A integração de canais digitais tem vindo a crescer nos últimos anos e as empresas devem adaptar-se a este fenómeno fortemente presente. Constituem uma porta para novas formas de comunicação e acesso a produtos/serviços ubiquamente (em qualquer lugar/ momento). Muitas empresas têm de repensar o seu modelo de negócio perante estas oportunidades/desafios para não serem meras expetadoras, mas sim agentes de mudanças sociais e económicas mais eficazes. Para isso, devem aprofundar a avaliação dos impactos da media social e suas tendências.

\section{Agradecimentos}

Este artigo é financiado por fundos da FCT - Fundação para a Ciência e Tecnologia, através do projeto UIDB/04020/2020.

\section{Referências}

Alméri, T. M., Mendes, A. d., Martins, L. F., \& Luglio, R. G. (2013). A influência das redes sociais nas organizações. Revista de Administração da Fatea, 7(7), 132-146.

Appel, G., Grewal, L., Hadi, R., \& Stephen, A. T. (2019). The future of social media in marketing. Journal of the Academy of Marketing Science, 48, 79-95.

Bala, M., \& Verma, D. (2018). A critical review of digital marketing. International Journal of Management, IT \& Engineering, 8(10), 321-339.

Cardoso, S. (2020). Google Ads - Adquira os clientes certos para o seu negócio. Webinar organizado pela NEST - Centro de Inovação do Turismo.

Ceyhan, A. (2019). The impact of perception related social media marketing applications on consumers' brand loyalty and purchase intention. Emerging Markets Journal, 9(1), 87-100.

Cruz, C. A., \& Silva, L. L. (2014). Marketing digital: Marketing para o novo milênio. Revista Científica do ITPAC, 7(2), 1-7. 
Fialho, J. M. (2014). Análise de redes sociais: Princípios, linguagem e estratégia de ação na gestão do conhecimento. Brasil: Editora Perspectivas em Gestão \& Conhecimento.

García, J. J., Lizcano, D., Ramos, C. M., \& Matos, N. (2019). Digital marketing actions that achieve a better attraction and loyalty of users: An analytical study. Future Internet, 11, 1-16.

Hysa, B., \& Spalek, S. (2019). Opportunities and threats presented by social media in project management. Heliyon, 5(4), 1-28.

Junusi, R. (2020). Digital marketing during the pandemic period; A study of Islamic perspective. Journal of Digital Marketing and Halal Industry, 2(1), 15-28. Doi: 10.21580/jdmhi.2020.2.1.5717.

Mason, A., Narcum, J. \& Mason, K. (2021). Social media marketing gains importance after Covid19. Cogent Business \& Management, 8(1), 1-17. Doi: 10.1080/23311975.2020.1870797.

Negocios SC (2020). A importância do marketing digital durante a pandemia, https://negociossc.com.br/blog/a-importancia-do-marketing-digital-durante-a-pandemia/

Pinheiro, J., Farias, T., \& Abe-Lima, J. (2013). Painel de especialistas e estratégia multimétodos: Reflexões, exemplos, perspectivas. Psico, 44 (2), 184-192.

Pourkhania, A., Abdipour, K., Bahera, B., \& Moslehpoura, M. (2019). The impact of social media in business growth and performance: A scientometrics analysis. International Journal of Data and Network Science, 3(3), 223-244.

Ramaswamy, V. (2008). Co-creating value through customers' experiences: The Nike case. Strategy \& Leadership, 36, 9-14. Doi: 10.1108/10878570810902068.

Sormaz, J., Kuzmanovic, M., \& Jeremic, V. (2019). Customer preferences towards digital marketing strategies. In book Economics of Digital Transformation, 507-526.

Souza, Q., \& Quandt, C. (2008). Metodologia de análise de redes sociais. In book O Tempo das Redes, 31-63.

Yamin, A. B. (2017). Impact of digital marketing as a tool of marketing communication: A behavioral perspective on consumers of Bangladesh. American Journal of Trade and Policy, 4(3), 49-54. 